\title{
Single-cell genomics of uncultured bacteria reveals dietary fiber responders in the mouse gut microbiota
}

\author{
Rieka Chijiwawa ${ }^{1,2+}$, Masahito Hosokawa ${ }^{3,4,5^{*+}}$ (D), Masato Kogawa ${ }^{1,2}$, Yohei Nishikawa ${ }^{1,2}$, Keigo Ide ${ }^{1,2}$, \\ Chikako Sakanashi ${ }^{3}$, Kai Takahashi ${ }^{1}$ and Haruko Takeyama ${ }^{1,2,3,4^{*}}$
}

\begin{abstract}
Background: The gut microbiota can have dramatic effects on host metabolism; however, current genomic strategies for uncultured bacteria have several limitations that hinder their ability to identify responders to metabolic changes in the microbiota. In this study, we describe a novel single-cell genomic sequencing technique that can identify metabolic responders at the species level without the need for reference genomes, and apply this method to identify bacterial responders to an inulin-based diet in the mouse gut microbiota.
\end{abstract}

Results: Inulin-feeding changed the mouse fecal microbiome composition to increase Bacteroides spp., resulting in the production of abundant succinate in the mouse intestine. Using our massively parallel single-cell genome sequencing technique, named SAG-gel platform, we obtained 346 single-amplified genomes (SAGs) from mouse gut microbes before and after dietary inulin supplementation. After quality control, the SAGs were classified as 267 bacteria, spanning 2 phyla, 4 classes, 7 orders, and 14 families, and 31 different strains of SAGs were graded as highand medium-quality draft genomes. From these, we have successfully obtained the genomes of the dominant inulin-responders, Bacteroides spp., and identified their polysaccharide utilization loci and their specific metabolic pathways for succinate production.

Conclusions: Our single-cell genomics approach generated a massive amount of SAGs, enabling a functional analysis of uncultured bacteria in the intestinal microbiome. This enabled us to estimate metabolic lineages involved in the bacterial fermentation of dietary fiber and metabolic outcomes such as short-chain fatty acid production in the intestinal environment based on the fibers ingested. The technique allows the in-depth isolation and characterization of uncultured bacteria with specific functions in the microbiota and could be exploited to improve human and animal health.

Keywords: Single-cell genomics, Gut microbiome, Uncultured bacteria, Mouse, Inulin, Bacteroides

\section{Background}

The gut microbiota plays a crucial role in the control of host physiology and metabolism through the release and transformation of metabolites like short-chain fatty acids (SCFAs) [1, 2]. Many studies have demonstrated that dietary fiber supplementation can modulate gut microbiota composition and promote SCFA production [3-5].

\footnotetext{
*Correspondence: m.hosokawa@aoni.waseda.jp; haruko-takeyama@waseda.jp Rieka Chijiwa and Masahito Hosokawa equally contributed to this work. ${ }^{3}$ Research Organization for Nano and Life Innovation, Waseda University, 513 Wasedatsurumaki-cho, Shinjuku-ku, Tokyo 162-0041, Japan

'Department of Life Science and Medical Bioscience, Graduate School of Advanced Science and Engineering, Waseda University, 2-2 Wakamatsucho, Shinjuku-ku, Tokyo 162-8480, Japan

Full list of author information is available at the end of the article
}

This dietary fiber metabolism is particularly important for non-digestible inulin-type fructans classified as prebiotics, which are substrates that promote the growth of beneficial microorganisms in the gut [6-12]. Previous reports indicated that several bacterial taxa in the intestine, including Firmicutes, Bacteroides, and Bifidobacterium, utilize fructan, and that dietary fructan can result in the growth of Actinobacteria, Firmicutes, or Bacteroides. However, our understanding of nutrient sensing and utilization by gut microbiota is limited, and there is a lack of predictability of the response of the microbiota to such dietary interventions. Thus, to enhance the benefits of dietary fiber to the host, it is important to identify inulin-responders in the complex microbiome community of the host intestine and 
characterize their mechanisms of inulin fermentation and potential for SCFA production.

Recently developed metagenomic approaches can provide comprehensive microbial profiling and putative functions using $16 \mathrm{~S}$ rRNA gene amplicon sequencing and shotgun metagenome sequencing. These methods have enabled the exploration of complex communities in the gut environment without the need for in vitro cultivation of the intestinal microbiome. However, 16S rRNA gene sequencing provides snapshots of microbial composition but does not provide information on microbial metabolic functions [13]. Shotgun metagenomics provides the complete genomic content of the microbial community, but it is generally difficult to link taxonomic and functional information for individual microbial strains [14]. Importantly, these techniques rely on the availability of reference microbial genomes for accurate functional assignments of specific organisms and precise taxonomic classification in the microbiome [15-17]. However, an estimated half of human gut species lack a reference genome. Thus, a new culture-independent genomic approach is required to enhance the reference genomes along with conventional metagenomic approaches and unveil the taxonomy and functions of the yet uncultured microbes at the strain level.

In this study, we used single-cell genome sequencing to identify microbial responders of dietary fiber, inulin, in mouse gut microbiota. Our massively parallel single bacterial genome sequencing technique, named singlecell amplified genomes in gel beads sequencing (SAGgel), utilizes a gel matrix that enables a serial enzymatic reaction for tiny genome amplification and provides support for the isolation of the amplified genomes. We targeted the family Bacteroidaceae from massively produced single-cell amplified genomes and characterized their polysaccharide utilization loci (PUL) and SCFA production pathways $[7,8,11]$.

Near-complete genomes obtained via single-cell genome sequencing provided us new insights into the mechanisms of microbial proliferation and SCFA production in the intestine. The resulting genome set can serve as the basis for strain-specific comparative genomics to understand uncultured organisms in the gut microbiome. These findings will help us to predict the metabolic fermentation of dietary fibers based on the presence and ability of the specific responders.

\section{Results}

\section{Effects of dietary fiber inulin on gut microbiota composition}

First, we assessed changes in mouse gut microbiota composition before and after inulin supplementation. To account for diurnal oscillations in gut microbiota composition $[18,19]$, feces were sampled in the morning and evening after 2 weeks of continuous inulin feeding. Cellulose, which is generally poorly fermented by gut microbes, was used as a control diet fiber. As shown in Fig. 1a, the mouse fecal microbiota mainly consisted of three classes, Bacteroidales, Lactobacillales, and Clostridiales, regardless of feeding conditions and sampling time. Lachnospiraceae and Streptococcaceae were dominant (25-30\% and $17-45 \%$, respectively) in the mice feces collected before fiber feeding and from the cellulose-fed mice, while in the feces from the inulin-fed mice, the abundance of Bacteroidaceae increased relative to other bacteria (17\% in morning and $41 \%$ in evening), while that of Lachnospiraceae decreased $(20 \%$ in morning and $12 \%$ in evening). These relative abundance profiles indicate a microbial composition increment of $\mathrm{Bac}$ teroidaceae in the feces of inulin-fed mice beyond typical diurnal oscillations in comparison to that in the cellulosefed mice ( $p$ values $<0.005$, Tukey's HSD test).

To identify the specific bacterial taxa associated with inulin fermentation, we compared the fecal microbiota of mice before fiber feeding and inulin-fed mice using the LDA effect size (LEfSe) analysis [20]. Differentially abundant taxa in the fecal microbiota and their predominant bacteria are shown in Fig. 1b, c. These results primarily indicated the diurnal differences, but revealed that Bacteroidales were abundant (LDA score $>2, p$ value $<0.05)$ in inulin-fed mice at both time points.

To confirm this effect, we monitored time-dependent microbiota composition changes in inulin-fed mice from another animal lot. The feces of inulin-fed mice were sampled in the morning for 2 weeks. As shown in Fig. 1d, Bacteroidaceae rapidly increased on day 1 , though there were individual differences in microbial composition. On days 3 to 5, Bacteroidaceae were most abundant in all mice, which were housed in three different cages. After this rapid increase, Bacteroidaceae gradually decreased but maintained their higher percentage in all mice except those in cage 1. In this lot of mice, we observed different microbiota compositional changes compared to the previous experiment, such as an increase in Bifidobacteriaceae in all cages, and in Akkermansiaceae in cage 1 during days 6 to 14. Despite differences in microbial composition between cages and animal lots, the results strongly suggest that Bacteroidaceae is a pivotal responder in the intestinal microenvironment of inulin-fed mice. In addition, we found two main Bacteroidaceae spp. (OTU2 and OTU4) in all mice, regardless of the fiber feeding condition. As shown in Additional file 2: Figure S1, OTU2 were rapidly increased after starting inulin feeding and showed significant compositional increment after 2 weeks of continuous inulin feeding ( $p$ values $<4 \mathrm{E}-02$, Tukey's HSD test). In contrast, OTU4 showed a constant composition during inulin feeding, while they showed significant increment compared to that in the cellulose-fed mice in the evening. 


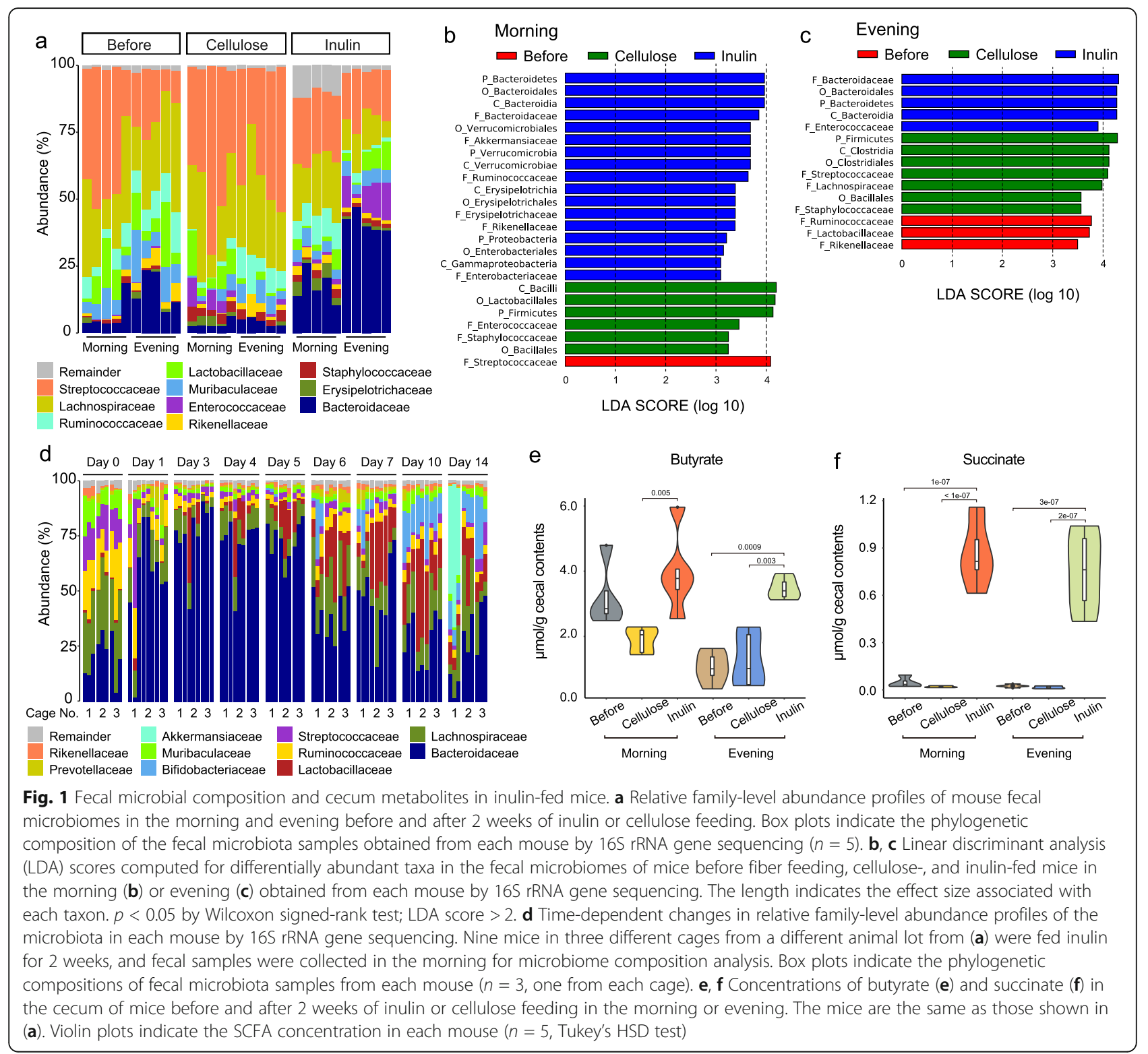

We also evaluated cecum SCFAs to observe the effects of inulin on gut microbiome metabolic function. Fig. 1e, $f$ shows the concentrations of butyrate and succinate in the cecum contents of mice used for 16S rRNA gene sequencing, measured by gas chromatography (GC). Although the total SCFA concentration depended on the diet and sampling time points in mice before fiber feeding and in the cellulose-fed mice (data not shown), samples from inulin-fed mice consistently contained higher butyrate and succinate concentrations.

Taken together, microbial composition and metabolome analyses suggest that inulin feeding changes the microbiome composition to increase Bacteroides spp. and microbial metabolic functions, resulting in increased succinate and butyrate concentrations in the mouse intestine.

\section{Single-cell resolution genome analysis of the fecal microbiome of inulin-fed mice}

Using the single-cell sequencing based on the SAG-gel platform (Fig. 2a), mouse intestinal microbes were captured in agarose gel beads by microfluidic droplet generator. Cell lysis with an enzyme cocktail, genomic DNA purification [21], and subsequent whole-genome amplification $[22,23]$ were all performed in the agarose gel matrix. After sorting the single beads, which contain single-cell amplified DNA, into standard PCR plates using a cell sorter, the DNA samples were re-amplified as a single-cell amplified genome (SAG) library. From 464 single-cell captured beads derived from mice fecal microbiota samples before (180 SAGs) and after inulin or cellulose feeding (166 SAGs), a total of 346 SAGs, 

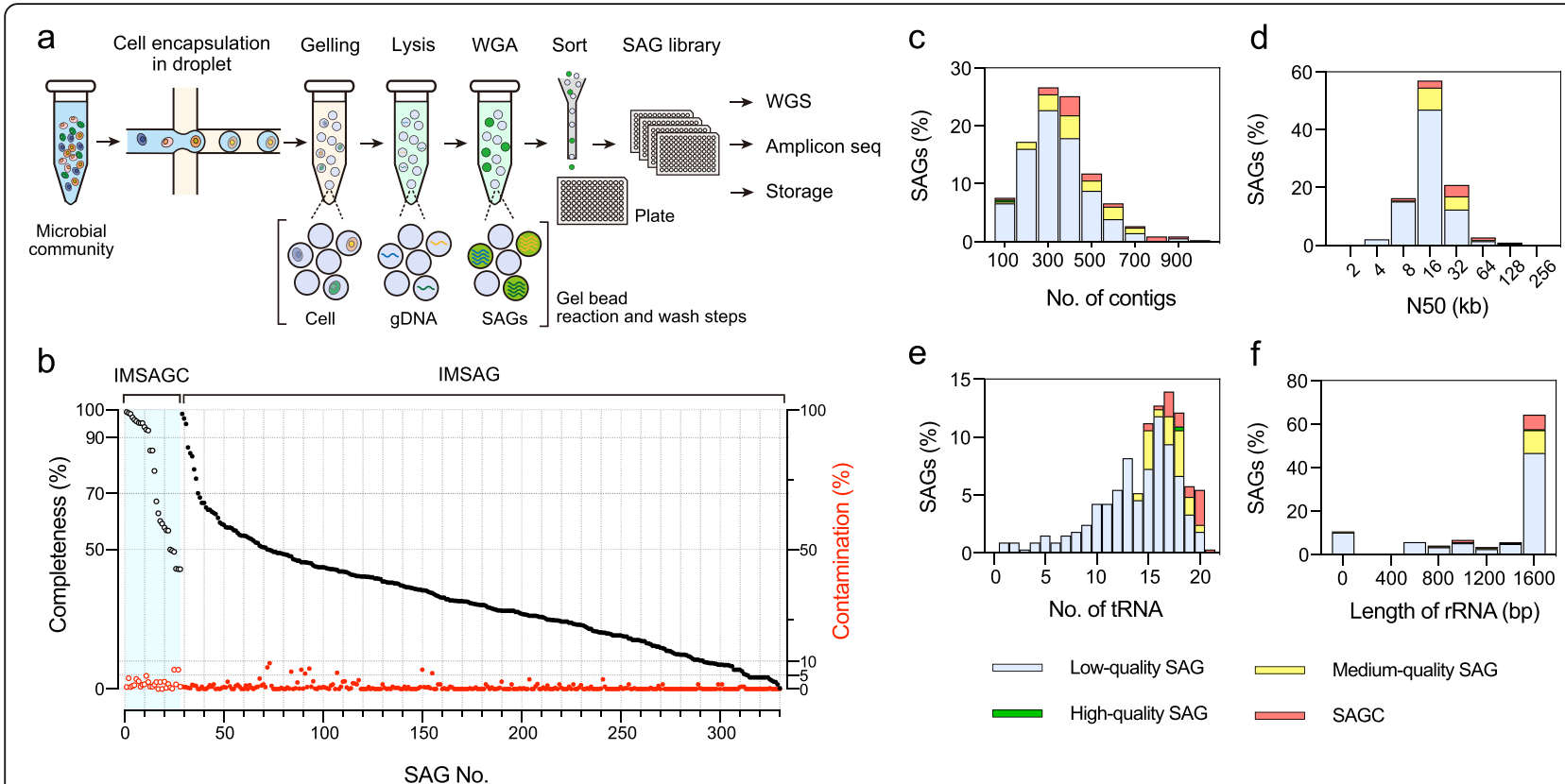

e

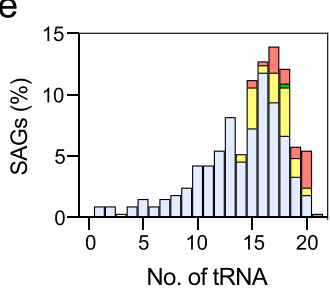

Low-quality SAG
High-quality SAG f

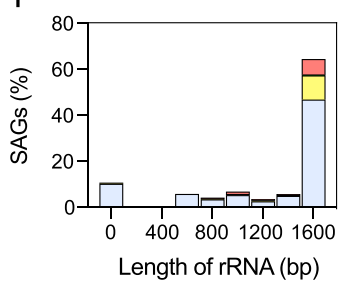

Medium-quality SAG

SAGC

Fig. 2 Single-cell genome sequencing of fecal microbiomes in inulin-fed mice.a Workflow for SAG-gel-based single-cell genome sequencing of bacterial cells in a complex microbial community. Individual bacterial cells are randomly captured in picoliter-sized gel beads and processed by in-gel lysis and whole-genome amplification (WGA). Single-cell amplified genomes (SAGs) in the gel are fluorescently detected and sorted into well plates as a SAG library. The SAGs are re-amplified for further analysis by NGS, amplicon sequencing, and storage. b-f Assembly qualities of 324 SAGs, excluding short fragments (length < $100 \mathrm{~kb}$ ) and contaminated samples (contamination > 10\%). b Completeness and contamination statistics for 302 SAGs (IMSAGs) and 22 composite SAGs (IMSAGCs). c-f The number of contigs, N50 values, number of tRNAs, and 16S rRNA gene lengths for 324 SAGs.

named IMSAG_001 to 346, were sequenced from the SAG library (Fig. 2b). According to the standards developed by the Genomic Standards Consortium [24], we recovered one high-quality draft genome, 43 medium-quality draft genomes, 258 low-quality draft genomes, 38 short genome fragments $(<100 \mathrm{~kb}$ or $0 \%$ completeness), and 6 contaminated genomes (Fig. 2b and Additional file 1: Table S1). The average completeness and contamination of the draft genomes were $31.8 \%$ and $0.6 \%$, respectively, with an average of 296 contigs (Fig. 2c). Notably, only 15 of the 346 (4.3\%) SAGs had $>5 \%$ contamination. Approximately $15 \%$ of SAGs were classified as high- or medium-quality draft genomes, ranging from 1.3-3.6 $\mathrm{Mb}$ in total contig length. The high- and medium-quality draft genomes had an average N50 of $16.4 \mathrm{~kb}$, while the low-quality draft genomes had an average of $10.4 \mathrm{~kb}$ (Fig. 2d). An average of $17 \mathrm{tRNAs}$ were detected in the high- and medium-quality draft genomes (Fig. 2e). Of the 302 SAG draft genomes, 267 (88\%) recovered the $16 \mathrm{~S}$ rRNA gene, $71 \%$ were $>1400 \mathrm{bp}$ in size (Fig. 2f), and 25\% contained an rRNA operon that encoded the 16S, 23S, and 5S rRNA genes in single contigs (Additional file 1: Table S1). When we conducted nucleotide BLAST searches against a database of $16 \mathrm{~S}$ rRNA gene sequences, 284 SAGs were similar to intestinal microbe $16 \mathrm{~S}$ rRNA gene sequences with $>80 \%$ identity, whereas 216 SAGs showed $<97 \%$ identity with the top search results, suggesting that many SAGs were obtained from uncultured bacteria that have not yet been registered in the database (Additional file 1: Table S2).

Qualified SAGs were classified as 267 bacteria, spanning 2 phyla, 4 classes, 7 orders, and 14 families with GTDB-Tk [25]. Of all samples, 123 (46\%) were classified as Lachnospiraceae, followed by Bacteroidaceae (65 (24\%)), and Muribaculaceae (27 (10\%); Additional file 1: Table S2). The phylogenetic distribution of these SAGs was slightly different from that of $16 \mathrm{~S} \mathrm{rDNA}$ genes acquired from a metagenomic sample, and the SAGs covered 62 of 298 operational taxonomic units (OTUs; 21\%; Additional file 1: Table S3). The SAGs covered seven major bacterial families in the metagenomic analysis (> $1 \%$ of the total OTUs in the inulin and cellulose samples) but missed the genomes of some gram-positive bacteria, such as Streptococcaceae, Enterococcaceae, and Staphylococcaceae. Moreover, some SAGs had strain marker gene identities $\geq 99 \%$, and average nucleotide identities (ANIs) $\geq 95 \%$ were co-assembled to improve their completeness as single strain genomes, which were named IMSAGC_001 to 028 (Additional file 1: Table S4). Finally, we obtained 31 different SAG strains (22 composite and 9 raw) that were graded as high- (7) or mediumquality (24) draft genomes among seven families (Table 1 and Additional file 1: Table S5). These included two 
Table 1 Statistics for high and medium quality draft genomes

\begin{tabular}{|c|c|c|c|c|c|c|c|c|c|c|}
\hline SAG ID & Lineage $^{a}$ & \# contigs & $\begin{array}{l}\text { Total } \\
\text { length } \\
\text { (Mb) }\end{array}$ & $\begin{array}{l}\text { Largest } \\
\text { contig } \\
\text { (bp) }\end{array}$ & $\begin{array}{l}\text { N50 } \\
\text { (bp) }\end{array}$ & $\begin{array}{l}\mathrm{GC} \\
(\%)\end{array}$ & $\begin{array}{l}\# \\
\text { CDS }\end{array}$ & $\begin{array}{l}\text { Completeness } \\
(\%)^{\mathrm{b}}\end{array}$ & $\begin{array}{l}\text { Contamination } \\
(\%)^{\mathrm{b}}\end{array}$ & $\begin{array}{l}\text { SAG } \\
\text { quality }^{c}\end{array}$ \\
\hline IMSAGC_001 & $\begin{array}{l}\text { Bacteroidota;Bacteroidia;Bacteroidales; } \\
\text { Bacteroidaceae;Bacteroides;Bacteroides } \\
\text { acidifaciens }\end{array}$ & 369 & 4.93 & 125378 & 29923 & 43.1 & 4165 & 97.27 & 1.12 & High \\
\hline IMSAGC_002 & $\begin{array}{l}\text { Firmicutes_A;Clostridia;Lachnospirales; } \\
\text { Lachnospiraceae;28-4 }\end{array}$ & 709 & 5.08 & 70754 & 13000 & 44.6 & 4725 & 93.87 & 1.78 & Medium \\
\hline IMSAGC_003 & $\begin{array}{l}\text { Firmicutes_A;Clostridia;Lachnospirales; } \\
\text { Lachnospiraceae;CAG-65 }\end{array}$ & 308 & 4.62 & 140873 & 48295 & 50.7 & 4203 & 98.85 & 3.97 & Medium \\
\hline IMSAGC_004 & $\begin{array}{l}\text { Bacteroidota;Bacteroidia;Bacteroidales; } \\
\text { Bacteroidaceae;Bacteroides_B }\end{array}$ & 305 & 4.22 & 106819 & 34968 & 43.1 & 3598 & 98.62 & 0.8 & Medium \\
\hline IMSAGC_005 & $\begin{array}{l}\text { Firmicutes_A;Clostridia;Lachnospirales; } \\
\text { Lachnospiraceae;CAG-95 }\end{array}$ & 317 & 4.23 & 113574 & 39279 & 43.6 & 4110 & 96.45 & 1.45 & Medium \\
\hline IMSAGC_006 & $\begin{array}{l}\text { Bacteroidota;Bacteroidia;Bacteroidales; } \\
\text { Muribaculaceae;UBA3263;GCA_ } \\
001689615.1\end{array}$ & 265 & 2.42 & 113083 & 24935 & 50.4 & 2284 & 85.47 & 0.89 & Medium \\
\hline IMSAGC_007 & $\begin{array}{l}\text { Firmicutes_A;Clostridia;Lachnospirales; } \\
\text { Lachnospiraceae }\end{array}$ & 496 & 5.06 & 122936 & 25158 & 47.1 & 4957 & 95.89 & 3.59 & Medium \\
\hline IMSAGC_008 & $\begin{array}{l}\text { Bacteroidota;Bacteroidia;Bacteroidales; } \\
\text { Muribaculaceae;CAG-873 }\end{array}$ & 380 & 2.66 & 59381 & 16733 & 52.1 & 2436 & 78.2 & 1.58 & Medium \\
\hline IMSAGC_009 & $\begin{array}{l}\text { Firmicutes_A;Clostridia;Lachnospirales; } \\
\text { Lachnospiraceae;CAG-95 }\end{array}$ & 597 & 5.02 & 113931 & 23340 & 44.4 & 4633 & 95.33 & 2.87 & Medium \\
\hline IMSAGC_010 & $\begin{array}{l}\text { Firmicutes;Bacilli;Lactobacillales; } \\
\text { Lactobacillaceae;Lactobacillus; } \\
\text { Lactobacillus johnsonii }\end{array}$ & 54 & 1.91 & 214598 & 104736 & 34.3 & 1789 & 99.22 & 0.78 & High \\
\hline IMSAGC_011 & $\begin{array}{l}\text { Firmicutes_A;Clostridia;Lachnospirales; } \\
\text { Lachnospiraceae;COE1 }\end{array}$ & 431 & 4.25 & 143151 & 27757 & 37.9 & 3664 & 92.66 & 2.59 & High \\
\hline IMSAGC_012 & $\begin{array}{l}\text { Firmicutes_A;Clostridia;Lachnospirales; } \\
\text { Lachnospiraceae;Eubacterium_J }\end{array}$ & 419 & 3.92 & 117373 & 25561 & 45.0 & 3748 & 92.92 & 4.75 & Medium \\
\hline IMSAGC_013 & $\begin{array}{l}\text { Firmicutes_A;Clostridia;Lachnospirales; } \\
\text { Lachnospiraceae;14-2 }\end{array}$ & 884 & 5.14 & 67964 & 11980 & 44.6 & 4945 & 85.42 & 0.95 & Medium \\
\hline IMSAGC_014 & $\begin{array}{l}\text { Bacteroidota;Bacteroidia;Bacteroidales; } \\
\text { Bacteroidaceae;F0040 }\end{array}$ & 273 & 2.64 & 87270 & 20006 & 47.1 & 2340 & 95.18 & 1.58 & Medium \\
\hline IMSAGC_015 & $\begin{array}{l}\text { Firmicutes_A;Clostridia;Lachnospirales; } \\
\text { Lachnospiraceae;Dorea }\end{array}$ & 388 & 2.55 & 63422 & 12891 & 45.3 & 2429 & 67.29 & 2.53 & Medium \\
\hline IMSAGC_016 & $\begin{array}{l}\text { Bacteroidota;Bacteroidia;Bacteroidales; } \\
\text { Muribaculaceae;CAG-485 }\end{array}$ & 385 & 1.94 & 50916 & 8558 & 46.4 & 1735 & 56.91 & 0.5 & Medium \\
\hline IMSAGC_017 & $\begin{array}{l}\text { Firmicutes;Bacilli;Erysipelotrichales; } \\
\text { Erysipelatoclostridiaceae; } \\
\text { Erysipelatoclostridium; } \\
\text { Erysipelatoclostridium cocleatum }\end{array}$ & 299 & 2.69 & 71470 & 19801 & 28.9 & 2384 & 95.28 & 0.94 & Medium \\
\hline IMSAGC_019 & $\begin{array}{l}\text { Firmicutes_A;Clostridia;Lachnospirales; } \\
\text { Lachnospiraceae;Dorea }\end{array}$ & 311 & 2.42 & 198629 & 20896 & 44.1 & 2370 & 62.98 & 0 & Medium \\
\hline IMSAGC_020 & $\begin{array}{l}\text { Firmicutes_A;Clostridia;Lachnospirales; } \\
\text { Lachnospiraceae;CAG-95 }\end{array}$ & 756 & 4.13 & 136678 & 11653 & 45.8 & 4150 & 57.89 & 2.63 & Medium \\
\hline IMSAGC_021 & $\begin{array}{l}\text { Bacteria;Firmicutes_A;Clostridia; } \\
\text { Lachnospirales;Lachnospiraceae;Dorea }\end{array}$ & 642 & 3.03 & 69412 & 9299 & 45.8 & 2954 & 60.15 & 2.58 & Medium \\
\hline IMSAGC_027 & $\begin{array}{l}\text { Bacteroidota;Bacteroidia;Bacteroidales; } \\
\text { Muribaculaceae;CAG-1031;GCA_ } \\
001689585.1\end{array}$ & 314 & 2.00 & 63820 & 15566 & 50.5 & 1738 & 59.25 & 0 & Medium \\
\hline IMSAGC_028 & $\begin{array}{l}\text { Bacteroidota;Bacteroidia;Bacteroidales; } \\
\text { Rikenellaceae;Alistipes }\end{array}$ & 265 & 1.73 & 77838 & 22105 & 52.5 & 1581 & 56.76 & 1.92 & Medium \\
\hline IMSAG_013 & $\begin{array}{l}\text { Firmicutes_A;Clostridia;Oscillospirales; } \\
\text { CAG-272 }\end{array}$ & 229 & 1.72 & 93908 & 16943 & 45.4 & 1622 & 59.65 & 0 & Medium \\
\hline IMSAG_025 & Bacteroidota;Bacteroidia;Bacteroidales; & 667 & 2.51 & 52448 & 6077 & 45.3 & 2558 & 50 & 7.76 & Medium \\
\hline
\end{tabular}


Table 1 Statistics for high and medium quality draft genomes (Continued)

\begin{tabular}{|c|c|c|c|c|c|c|c|c|c|c|}
\hline SAG ID & Lineage $^{a}$ & \# contigs & $\begin{array}{l}\text { Total } \\
\text { length } \\
\text { (Mb) }\end{array}$ & $\begin{array}{l}\text { Largest } \\
\text { contig } \\
\text { (bp) }\end{array}$ & $\begin{array}{l}\text { N50 } \\
\text { (bp) }\end{array}$ & $\begin{array}{l}\mathrm{GC} \\
(\%)\end{array}$ & \# & $\begin{array}{l}\text { Completeness } \\
(\%)^{b}\end{array}$ & $\begin{array}{l}\text { Contamination } \\
(\%)^{\mathrm{b}}\end{array}$ & $\begin{array}{l}\text { SAG } \\
\text { quality }^{c}\end{array}$ \\
\hline & Muribaculaceae;CAG-485 & & & & & & & & & \\
\hline IMSAG_044 & $\begin{array}{l}\text { Firmicutes;Bacilli;Lactobacillales; } \\
\text { Lactobacillaceae;Lactobacillus_- } \\
\text { H;Lactobacillus_H reuteri_A }\end{array}$ & 200 & 1.82 & 108153 & 17958 & 38.3 & 1735 & 86.54 & 0.54 & Medium \\
\hline IMSAG_049 & Firmicutes_A;Clostridia;Lachnospirales & 317 & 1.74 & 50322 & 11294 & 39.6 & 1762 & 54.88 & 1.34 & Medium \\
\hline IMSAG_117 & $\begin{array}{l}\text { Firmicutes;Bacilli;_Lactobacillales; } \\
\text { Lactobacillaceae;Lactobacillus_B; } \\
\text { Lactobacillus_B murinus }\end{array}$ & 314 & 1.86 & 38237 & 10806 & 40.5 & 1824 & 78.75 & 1.31 & Medium \\
\hline IMSAG_185 & $\begin{array}{l}\text { Firmicutes_A;Clostridia;Lachnospirales; } \\
\text { Lachnospiraceae;CAG-65 }\end{array}$ & 317 & 2.25 & 78895 & 17758 & 49.9 & 2117 & 54.01 & 1.45 & Medium \\
\hline IMSAG_192 & $\begin{array}{l}\text { Bacteroidota;Bacteroidia;Bacteroidales; } \\
\text { Muribaculaceae;Muribaculum; } \\
\text { Muribaculum intestinale }\end{array}$ & 342 & 1.89 & 59599 & 11500 & 49.3 & 1711 & 57.55 & 0 & Medium \\
\hline IMSAG_249 & $\begin{array}{l}\text { Firmicutes_A;Clostridia;Lachnospirales; } \\
\text { Lachnospiraceae;CAG-95 }\end{array}$ & 428 & 2.83 & 108242 & 17278 & 44.4 & 2659 & 55.18 & 0.91 & Medium \\
\hline IMSAG_250 & $\begin{array}{l}\text { Firmicutes_A;Clostridia;Oscillospirales; } \\
\text { DTU089;Eubacterium_R }\end{array}$ & 147 & 1.35 & 87509 & 28237 & 37.8 & 1309 & 68.68 & 0 & Medium \\
\hline
\end{tabular}

a Assigned using GTDB-Tk

${ }^{\mathrm{b}}$ Estimated using CheckM

c Classified according to GSC MISAG

Bacteroidaceae spp. (IMSAGC_001 and IMSAGC_004) that had identical 16S rRNA genes to Bacteroidaceae (OTU2 and OTU4, respectively), which were potential inulin-responders in inulin-fed mice feces (Additional file 1: Tables S3 and S5, and Additional file 2: Figure S1).

\section{Genomic features of Bacteroides spp. draft genomes}

Bacteroides spp. are capable of metabolizing fructans, a class of plant and microbial polysaccharides $[7,9,11]$. The completeness of the inulin responder Bacteroides draft genomes IMSAGC_001 and IMSAGC_004 were sufficiently high $(\geq 97 \%)$ for comparison with publicly available genomes of Bacteroides spp. known to be fructan utilizers or showing similarity to these genomes (Additional file 1: Table S6 and Fig. 3a). Both had $>4.2 \mathrm{Mb}$ genome sizes, $43 \%$ GC content, and $>3500$ coding sequences (CDSs), similar to the genomes of Bacteroides spp. including $B$. thetaiotaomicron (Bt), B. ovatus (Bo), B. caccae (Bc), $B$. vulgatus (Bv), B. uniformis (Bu), B. fragilis (Bf), and $B$. acidifaciens (Ba; Fig. $3 \mathrm{a}$ and Table S6). Genes in these Bacteroides groups were clustered into 5013 different orthogroups. The orthogroup distributions between different Bacteroides group members are shown in Fig. 3b; of these orthogroups, 1150 were defined as core orthogroups. IMSAGC_001 and IMSAGC_004 shared a number of different orthogroups with specific strains, such as $\mathrm{Ba}$ (196 orthogroups) and $\mathrm{Bv}$ (99 orthogroups), consistent with the phylogenetic analysis (Fig. 3a). Although the vast majority of the orthogroups (151 and 57 in IMSAGC_001 and IMSAGC_004, respectively) were classified as hypothetical proteins of unknown function, most orthogroups shared between IMSAGC_001 and Ba belonged to category $\mathrm{O}$ (carbohydrate transport and metabolism; nine orthogroups), followed by categories $\mathrm{T}$ and $\mathrm{P}$ (signal transduction mechanisms and inorganic ion transport and metabolism, respectively; six orthogroups each). In addition, IMSAGC_001 and IMSAGC 004 shared 47 unique orthogroups (37 with unknown functions).

\section{Identification of putative polysaccharide utilization loci from Bacteroides spp. draft genomes}

Most of the glycan degrading and import machinery within Bacteroides genomes are encoded within clusters of coregulated genes known as polysaccharide utilization loci (PUL). The defining characteristic of a PUL is the presence of a pair of genes homologous to susD and sus $C$, which encode outer membrane proteins that bind inulin and import its digestion products $[8,11,26]$. The susC and susD homologs are usually associated with genes encoding the machinery necessary to convert extracellular polysaccharides into intracellular monosaccharides, such as glycoside hydrolases (GHs) or polysaccharide lyases (PLs). In addition, most PULs contain or are closely linked to genes encoding an inner membrane-associated sensor-regulator system, including the hybrid two-component system. Notably, some $\mathrm{Bac}$ teroides species, such as $\mathrm{Bc}, \mathrm{Bo}, \mathrm{Bt}$, and $\mathrm{Bu}$, can utilize inulin with similar efficiency to glucose $[7,8,11]$, while $\mathrm{Bv}$ lacks susC- and susD-like genes within its fructan PUL and cannot use inulin. Bt and Bo encode a number of predicted signal peptidase (SP)II-containing GHs/PLs 


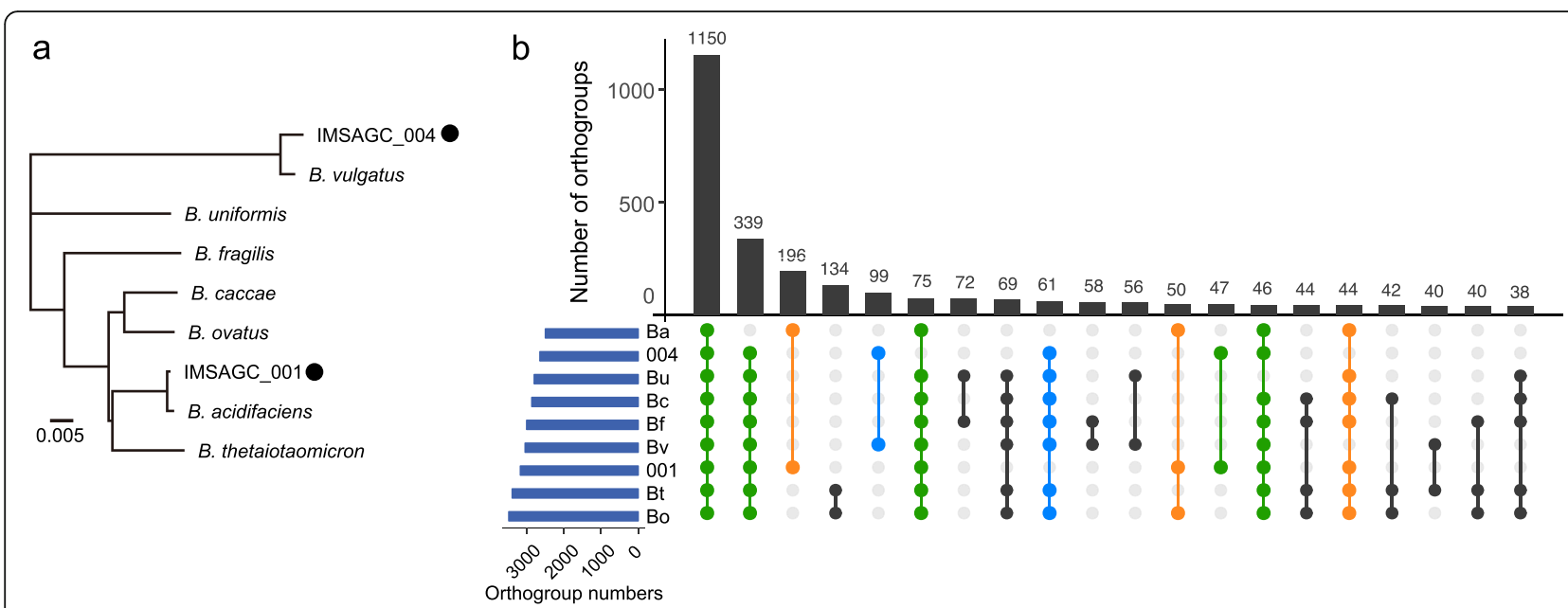

Fig. 3 Comparative analysis of the inulin responder Bacteroides genomes IMSAGC_001 and IMSAGC_004. a Phylogenetic tree of inulin-associated Bacteroides spp. found in this study and reference Bacteroides strains. The tree is based on the alignment of concatenated amino acid sequences of collocated sets of ubiquitous, single-copy genes within a phylogenetic lineage, as used in CheckM analysis. IMSAGC_001 and IMSAGC_004 are indicated by black circles. $\mathbf{b}$ Upset plot comparing shared orthogroup counts between reference Bacteroides strains. Orthogroups shared between IMSAGC_001 and other Bacteroides group members are colored in orange, whereas orthogroups shared between IMSAGC_004 and other Bacteroides group members are colored in blue. Orthogroups shared between IMSAGC_001, IMSAGC_004, and other members are colored in green. Bt, B. thetaiotaomicron; Bo, B. ovatus; BC, B. caccae; Bv, B. vulgatus; $\mathrm{Bu}, \mathrm{B}$. uniformis; Bf, B. fragilis; $\mathrm{Ba}$, B. acidifaciens

and act as public goods producers in various networks of polysaccharide utilizers [7]. These producer-derived GHs are extracellularly transported to create polysaccharide degradation products that can be utilized for the growth of concomitant recipients, such as $\mathrm{Bv}$, at a considerable distance from the producer.

We detected PULs containing inulinases in two Bacteroides draft genomes (IMSAGC_001 and IMSAGC_ 004; Fig. 4a, b). We also identified other Bacteroidales, such as IMSAGC_014 and IMSAGC_006, that had no PULs encoding inulinases. At the whole genome level, IMSAGC_001 showed similarity to inulin utilizers such as Bo, Bc, and Bt, while IMSAGC_004 was similar to the inulin non-utilizer Bv (Fig. 3a). In IMSAGC_001, the sequences of $\mathrm{GH}$ genes, including inulinase, showed high similarity to those of $\mathrm{Bc}$ and $\mathrm{Bo}$, which have superior ability to use inulin $[7,11]$ and had the highest similarity to Ba (Fig. 4c). IMSAGC_004 also contained a GH gene similar to that of known inulin utilizers. The GHs of IMSAGC_001 and IMSAGC_004 are predicted to be periplasmic, as they contain SPI cleavage sites. In addition, IMSAGC_001, IMSAGC_004, and Ba have no predicted SPII-containing GHs and PLs in their inulin utilization loci (Fig. 4b). Since Bacteroides spp. show differences in numbers of GH/PL-encoding genes and uniformity among strains of a given species [7], we compared the number of SPII-containing GH/PLs among Bacteroides genomes. IMSAGC_001 had a medium level of SPIIcontaining GH/PLs (56, Additional file 1: Table S7), while IMSAGC_004 and Ba had extremely low levels of SPIIcontaining GH/PLs (26 and 32, respectively). This result indicated that IMSAGC_001 and IMSAGC_004 might contain different types of machinery for polysaccharide utilization from Bt and Bo, which have several SPIIcontaining GH/PLs, which act as producers for a larger repertoire of polysaccharides.

For susC/D homologs, IMSAGC_001 and IMSAGC_ 004 clustered with $\mathrm{Ba}$ and apart from other inulin utilizers (Bc, Bo, Bu, and Bt; Fig. 4d). Homologs of the SusC and SusD outer membrane proteins are the defining features of the Sus-system, and genes encoding these proteins are found as a pair in most Bacteroidetes genomes. Inulin chains that may or may not be extracellularly cleaved are threaded through the SusC porin, and all degradation occurs in the periplasm. In Bo, deletion of the susC and susD orthologs results in significant growth impairment on inulin, and the orthologs are predicted to function in inulin-binding and import without prior extracellular digestion $[9,26]$. As our data suggested that IMSAGC_001 and IMSAGC_004 had low similarity to the Bo susC/D homologs, we next compared genome sequence similarities through structure prediction-based analysis. Based on structure prediction, the susC/D homologs in IMSAGC_001 and IMSAGC_004 could form complex structures composed of homodimers of an extracellular SusD-like lipoprotein and an integral membrane SusC-like transporter (Additional file 2: Figure S2). These predicted structures showed high similarity to the $\mathrm{Ba}$ complex at the amino acid level (Additional file 2: Figure S2).

In summary, Bacteroides IMSAGC_001, which were significantly increased in inulin-fed mouse feces, and 


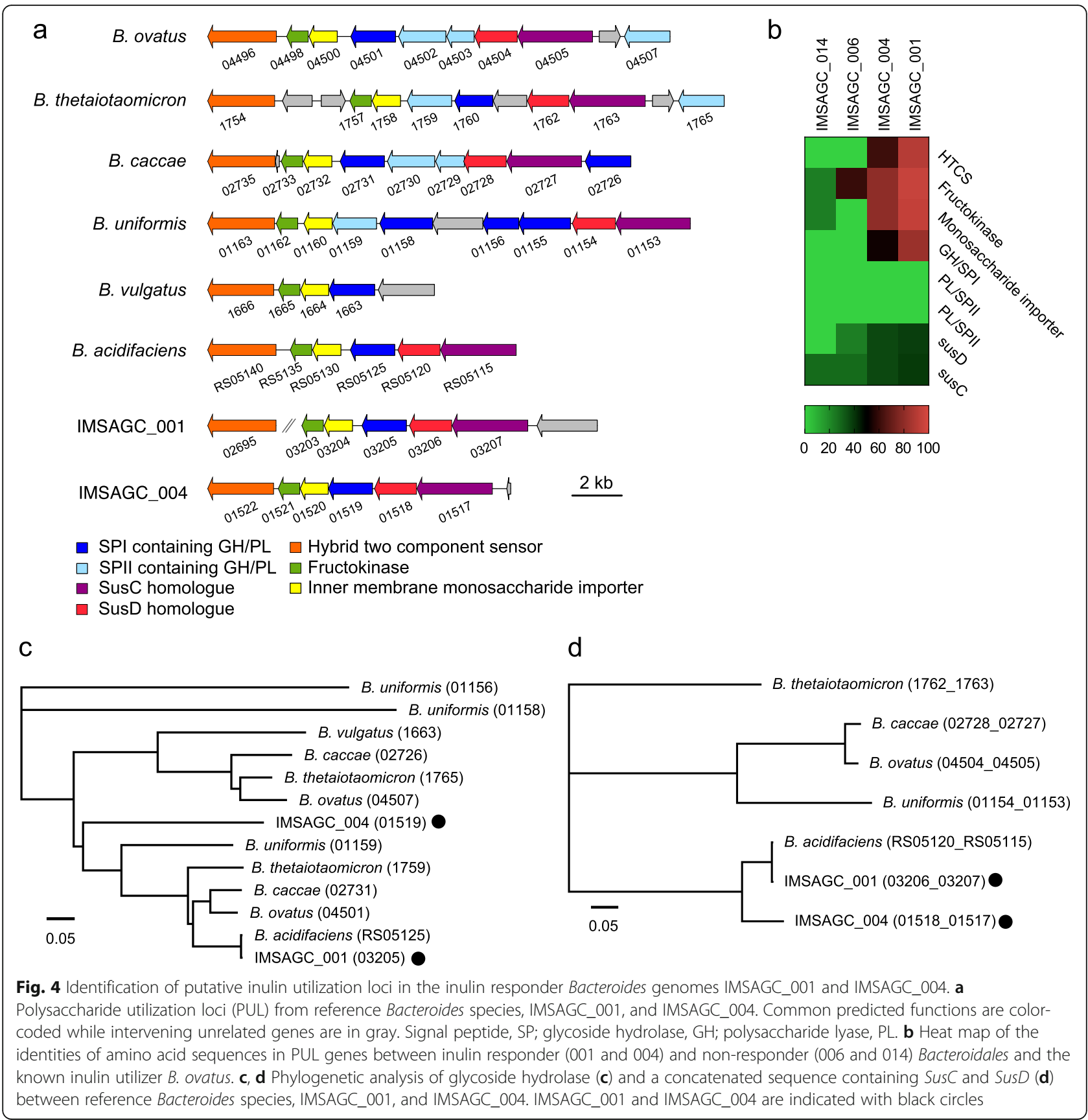

IMSAGC_04, which showed no apparent changes, possess putative PULs that include $s u s C / D$ homolog pairs, a putative outer membrane lipoprotein, a putative inner membrane monosaccharide importer, a putative fructokinase, and a putative inulin-specific $\mathrm{GH}$. No other SAGs had higher similarity to Bacteroides PUL. We predict that these bacteria import inulin directly via SusC and SusD for degradation to monosaccharides in the periplasm without prior extracellular degradation.
Identification of metabolic characters in inulin-utilizing strains and other bacterial SAGs

Having identified key responder bacterial strains for inulin metabolism, we next examined their fundamental metabolic functions in the mouse gut microbiome. Additional file 1: Tables S8 and S9 summarize the metabolic functions of all high- and medium-quality genomes obtained in this study (IMSAGC series with $>80 \%$ completeness). Notably, inulin-fed mice displayed significant increases in Bacteroides in their microbial composition 
and succinate and butyrate concentrations in their SCFAs. For succinate, we focused on the reductive tricarboxylic acid (TCA) cycle, as it produces the major end product of energy metabolism in anaerobic microorganisms. Many bacteria have incomplete TCA cycles and are commensal obligate anaerobes, including Bacteroides spp., which use a branched TCA cycle to support fumarate respiration $[27,28]$. The end product of fumarate reduction, succinate, is secreted into the extracellular environment. In IMSAGC_001 and IMSAGC_004, we detected reductive and incomplete TCA cycles from oxaloacetate to succinate (Additional file 2: Figure S3 and Additional file 1: Table S8), while no other predominant SAG contained both an inulin PUL and a succinate pathway.

In single-cell genome sequencing, the profiling of SAG metabolic pathways provides various insights into the traits of diverse microbiota at once, because each metabolic gene is specifically assigned to specific bacterial strains (Additional file 1: Table S9). As an interesting functional signature, we detected the presence of antibiotic resistance modules, such as vancomycin resistance, D-Ala-D-Ser type, in specific Lachnospiraceae strains (IMSAGC_003 and IMSAGC_007). We observed differences in conserved biosynthetic pathways in cofactor and vitamin metabolism, such as biotin and cobalamin synthesis, among different intestinal microbes. Comparing IMSAGC_001, IMSAGC_004, and other Bacteroides spp., IMSAGC_004 lacks proline and tryptophan biosynthesis pathways, as well as the first carbon oxidation pathway in the TCA cycle, but has a conserved cobalamin biosynthetic pathway similar to those of $\mathrm{Bf}$, $\mathrm{Bu}$, and Bv. Both IMSAGC_001 and IMSAGC_004 lack glycine betaine/proline transport systems, similar to $\mathrm{Ba}$ and $\mathrm{Bu}$. The inulin non-responsive Bacteroidales spp. (IMSAGC_014 and IMSAGC_006) lacked some carbohydrate metabolism pathways and saccharide transport systems compared to known polysaccharide utilizers. These results suggest that inulin-responsive Bacteroides IMSAGC_001, IMSAGC_004, and inulin non-responder Bacteroidales spp. play different metabolic roles in the mouse gut microbiota.

\section{Discussion}

Inulin ( $\beta 2-1$ fructan) is resistant to host-mediated digestion, resulting in remarkable fecal microbiome changes and cecum SCFA concentrations through intestinal microbial fermentation. In this study, to elucidate the inulin-responders in mouse intestine microbiota, we conducted 16S rRNA gene sequencing for the assessment of changes in the inulin-dependent microbiota composition, metabolome analysis for the evaluation of SCFA production, and single-cell genome sequencing for obtaining the draft genomes of inulin-responders.
The microbiome composition and SCFA amounts displayed typical diurnal oscillations, and inulin intake resulted in significant changes beyond these. Bacteroides spp. are known to utilize levan and inulin (Figs. 1 and 3, Additional file 1: Table S6). Microbiome composition monitoring demonstrated rapid responses by Bacteroides IMSAGC_001 to inulin supplementation and dynamic daily changes. However, reference Bacteroides strains have different origins and uncultured strains present in individual hosts could have different metabolic features. Bacteroides members have differing abilities to use these various plant polysaccharides. Species that utilize a particular polysaccharide (producers), such as Bo, liberate polysaccharide degradation products that are consumed by other recipients unable to grow using the intact polysaccharide $[7,8]$.

Functional characterization of bacteria using 16S rRNA gene sequencing databases may lead to misinterpretations. From a partial 16S rRNA gene sequence, responder bacteria can only be identified at the family level, Bacteroidaceae, and insights on the inulin utilization abilities of specific bacteria in the diverse microbial community cannot be obtained (Fig. 1 and Additional file 1: Table S3). Therefore, single-cell sequencing was used to analyze closely related species and compare uncultured bacterial genomes to identify inulin-responders in complex microbial communities.

The advantage of single-cell sequencing with the SAGgel platform is that it can select specific sequencing samples after generating large numbers of SAGs. Since we can adjust the total sequencing effort according to the number of SAGs required, it is not necessary to produce a large number of reads to cover target bacteria with the same depth as in shotgun metagenomic sequencing. Since each SAG is composed of tens of thousands of reads on average, limiting the analysis to specific strains enables extremely cost-efficient whole-genome sequencing of target cells. Indeed, approximately half of the SAGs were obtained from up to 96-plex indexes in a single sequence runs by MiSeq (total $\sim 3 \mathrm{~Gb}$ ). This prescreening can utilize marker genes, such as 16S rRNA genes, to select Bacteroidaceae and other abundant strains based on initial 16S rRNA gene sequencing. Even if the target bacteria were selected from the results of initial $16 \mathrm{~S}$ rRNA gene sequencing, it is challenging to demonstrate direct agreement between the MAGs and the target bacteria as most MAGs lack the rRNA and tRNA sequences in the contigs [15-17]. Thus, SAGs with functional and phylogenetic information are useful in combination with stepwise multilayer analyses, including screening through $16 \mathrm{~S}$ rRNA gene sequencing, to achieve the strategic genomic analysis of target bacteria.

Conversely, nearly $90 \%$ of the SAGs obtained in this study contained $16 \mathrm{~S}$ rRNA gene sequences, $70 \%$ of 
which were full-length, and many existed in operons with the $5 \mathrm{~S}$ and $23 \mathrm{~S}$ rRNA genes. The SAGs provide the genetic information of dominant microbes by covering dominant OTUs existing in $>1 \%$ of the fecal microbiome community. However, there is still room for improvement. For example, modifying the cell lysis procedure could enhance the variety of SAGs detected, including gram-positive bacteria. In $16 \mathrm{~S}$ rRNA gene homology analysis, a considerable number of SAGs showed low similarity to reference genomes, suggesting that these SAGs contain the genomes of species currently undescribed in public databases. Furthermore, by acquiring duplicate SAG data from identical strains, we could assemble the draft genomes using a cleaning and co-assembly strategy [22, 23]. For example, IMSAGC_ 001 and IMSAGC_004 were duplicated 41 and 12 times, respectively, allowing the construction of composite draft genomes, which are very advantageous for obtaining genomes of medium or high quality. These two main Bacteroides spp. were present in all mice, regardless of inulin feeding (Additional file 1: Table S3). Thus, we utilized raw SAGs for assembly improvement from all mice regardless of fiber feeding conditions. According to the definition used in Almeida et al. [16], 40\% of the draft genomes of quality higher than medium were qualified as near-complete ( $>90 \%$ completeness and $<5 \%$ contamination, Additional file 1:Table S5). Hence, the SAGgel platform can provide increased numbers of draft genomes from single microbial cells, with higher completeness than other single-cell genome sequencing techniques [22, 23, 29-32]. The ratio of high- and medium-quality draft genomes was comparable with recent metagenomic assembly and binning strategies for obtaining MAGs as draft genomes [15-17].

Pan-genome analysis within the same environment reveals what bacteria are present and which harbor specific genes (Additional file 1:Table S9). Our results revealed PUL and succinate synthesis pathways in inulin responder Bacteroidaceae. In addition, comparative SAG analysis allowed us to understand the conservation of various pathways, such as those involved in antibiotic resistance and vitamin synthesis, in each bacterial strain in the intestinal microbiome. Single strain-resolution fingerprinting of the microbiome enables the identification of bacteria or genes responsible for specific metabolic function from a complex microbial community. In this study, two responder Bacteroides genomes had inulinutilizing PUL clusters that showed similarity to known inulin utilizers. Our genome analyses suggested that Bacteroides IMSAGC 001 and IMSAGC 004, which can take up and degrade inulin, grow predominantly in the intestines of inulin-fed mice, and produce succinate through the glycolytic system and a reductive partial TCA cycle for release into the intestinal environment.
We have identified the potential inulin utilizers Bacteroides IMSAGC_001 and IMSAGC_004 in uncultured fecal microbiomes as a new subgroup with $\mathrm{Ba}$. Ba was originally isolated from mouse cecum as two strains that differed in their starch hydrolysis functions [33]. Their major end products of glucose fermentation are acetate and succinate, similar to those predicted for Bacteroides IMSAGC_001 and IMSAGC_004. Our data suggest that Bacteroides IMSAGC_001 and IMSAGC_004 utilize inulin for their metabolism and lack the ability to liberate the fructose for other species in the same manner as $\mathrm{Bf}$ and $\mathrm{Ba}$ [7]. The significant increase in Bacteroides IMSAGC_001 over IMSAGC_004 (Additional file 2: Figure S1) may be due to differences in the cell growth rate, as well as inulin uptake and degradation rates, given the differences in GHs and other PUL genes.

\section{Conclusion}

In this study, single-cell genomics enabled detailed microbiome analysis and identified inulin-responders with putative roles in dietary fiber metabolism. The technique generates a massive preparation of SAGs, enabling the functional analysis of uncultured bacteria in the intestinal microbiome and the consideration of host responses. The analysis makes it possible to estimate metabolic lineages in bacterial PUL and metabolic outcomes in the intestinal environment, such as SCFA production, based on ingested fibers. Genetic and functional differences between intestinal bacterial species are predictive of in vivo competitiveness in the presence of dietary fibers. Taxonomic and functional genes regulating bacterial metabolic capacity can serve as potential biomarkers in microbiome datasets and can be sequenced to assess microbiota control via diet. In the future, it may be possible to estimate responders in advance based on the presence of these microorganisms and genes.

\section{Methods \\ Mice}

Wild-type 6-week-old male mice from the BALB/C background (Tokyo Laboratory Animals Science Co., Japan) were used. All mice were housed in a 12:12 light:darkness cycle (Zeitgeber time $(\mathrm{ZT}) 0=8 \mathrm{AM}, \mathrm{ZT}$ $12=8 \mathrm{PM})$. Before feeding experiments, the mice were fed AIN93M (Oriental Yeast Co., Japan) for 1 week. After that, the mice were fed inulin- or cellulosesupplemented diets for two weeks in the morning or evening. To prepare the fiber-supplemented feed, AIN93M powder was mixed with $5 \%$ inulin or $5 \%$ cellulose, and then pellets were formed with tap water. Fecal samples were collected for $16 \mathrm{~S}$ rRNA gene sequencing and single-cell sequencing, and cecum samples were harvested before and after the 2-week fiber 
feeding for metabolomic analysis. All samples were stored at $-80^{\circ} \mathrm{C}$.

\section{DNA extraction from fecal samples}

Fecal samples (200 mg) were homogenized with $20 \mathrm{~mL}$ phosphate-buffered saline (PBS) with a Pellet Pestle ${ }^{\mathrm{rm}}$ Cordless Motor (Fisher Scientific). The fecal suspensions were then filtered through a $100-\mu \mathrm{m}$ nylon mesh to remove eukaryotic cells and other debris, and the flow-through suspensions were collected as the bacterial cell fraction. Filtered fecal suspensions were then centrifuged at $9000 \times g$ for $10 \mathrm{~min}$ at $4{ }^{\circ} \mathrm{C}$, and the bacterial pellets were resuspended in $800 \mu \mathrm{L}$ Tris-ethylenediaminetetraacetic acid (TE) 10 buffer (10 mM Tris-HCl/10 mM EDTA). Bacterial pellets were serially incubated with $100 \mu \mathrm{L}$ lysozyme $(150 \mathrm{mg} /$ $\mathrm{mL})$ at $37^{\circ} \mathrm{C}$ for $1 \mathrm{~h}, 20 \mu \mathrm{L}$ achromopeptidase $(100 \mathrm{U} / \mu \mathrm{L})$ at $37{ }^{\circ} \mathrm{C}$ for $30 \mathrm{~min}$, and $50 \mu \mathrm{L}$ proteinase $\mathrm{K}(20 \mathrm{mg} / \mathrm{mL})$ with $50 \mu \mathrm{L} 20 \%$ sodium dodecyl sulfate (SDS) at $55^{\circ} \mathrm{C}$ for 1 $\mathrm{h}$. The lysates were then treated with equal volumes of phenol:chloroform:isoamyl alcohol and centrifuged at $6000 \times g$ for $10 \mathrm{~min}$. After centrifugation, DNA in the upper aqueous layer was transferred to another tube and then pelleted by adding 3M sodium acetate solution and isopropanol, followed by centrifugation at $6000 \times g$ for $10 \mathrm{~min}$. DNA pellets were rinsed with 70\% ethanol, dried, and dissolved in TE buffer (10 mM Tris-HCl/1 mM EDTA). The collected DNA samples were purified with $10 \mu \mathrm{g} / \mathrm{mL}$ RNase and incubated at $37{ }^{\circ} \mathrm{C}$ for $30 \mathrm{~min}$ and precipitated by adding equal volumes of $20 \%$ polyethylene glycol solution (PEG6000). After centrifugation at $10,000 \times g$ for $10 \mathrm{~min}$ at 4 ${ }^{\circ} \mathrm{C}$. DNA pellets were rinsed with $70 \%$ ethanol, dried, and dissolved in TE buffer. The extracted DNA samples were stored at $-20^{\circ} \mathrm{C}$.

\section{S rRNA gene sequencing and analysis}

The V3-V4 hypervariable regions of 16S rRNA genes were analyzed according to the Illumina protocol for 16S Metagenomic Sequencing Library Preparation. Barcoded amplicons, amplified with 341F and 806R primers (Forward, 5' -TCGTCGGCAGCGTCAGATGTGTATAA GAGACAGCCTACGGGNGGCWGCAG-3'; reverse, 5' -GTCTCGTGGGCTCGGAGATGTGTATAAGAGAC AGGACTACHVGGGTATCTAATCC-3') were sequenced using the Illumina MiSeq $2 \times 300$ bp platform with MiSeq Reagent Kit v3 (Illumina Co.) according to the manufacturer's instructions. Raw reads were processed using QIIME2 v.2019.1 [34] using the DADA2 [35] plugin to denoise quality filter reads, call amplicon sequence variants (ASVs), and generate a feature table of ASV counts and host metadata. In the quality filtering step, the datasets were truncated to read length of 270 to 250 base pairs for the forward and reverse reads (all other parameters were set to default values). After quality filtering, bacterial taxonomies were assigned to the
ASV feature table using the Naïve Bayesian Q2 feature classifier as implemented in QIIME2. We compared the data against a SILVA reference database [36] trained on the V3-V4 region of the 16S rRNA gene.

Merged paired-end sequence reads of each sample were trimmed from the primer sequences, and lowquality reads (base error rate $>1 \%$ ) and chimeric sequences were removed using USEARCH [37]. Filtered sequence reads were assigned to operational taxonomic units (OTUs) by open-reference OTU picking at 97\% identity using UPARSE algorithm [38] against a SILVA reference database [36]. Taxonomic assignment of the reference sequence was used as the taxonomy for each OTU, and taxonomy table at the family level was generated.

LEfSe analysis was performed to identify taxa displaying the largest differences in microbiota abundance between groups [20]. Only taxa with LDA scores $>2.0$ and $p<0.05$, as determined by Wilcoxon signed-rank test, are shown. All data analyses were performed using $\mathrm{R}$ software v3.5.3.

\section{Metabolome analysis from cecum samples}

SCFA concentrations of cecum contents were determined by GC-mass spectrometry. In brief, $50 \mathrm{mg}$ cecum contents were homogenized with ether. After vigorous shaking using a Shake Master Neo (Bio-Medical Science) at $1500 \mathrm{rpm}$ for $10 \mathrm{~min}$, homogenates were centrifuged at $1000 \times g$ for $10 \mathrm{~min}$, then the top ether layers of each sample were collected and transferred to new glass vials. Aliquots of the ether extracts $(80 \mu \mathrm{L})$ were mixed with $16 \mathrm{~mL} \mathrm{~N}$-tert-butyldimethylsilyl-N-methyltrifluoroacetamide. The vials were sealed tightly and heated at $80{ }^{\circ} \mathrm{C}$ for $30 \mathrm{~min}$ in a water bath. Derivatized samples were loaded onto a 6890N Network GC System (Agilent Technologies) equipped with an HP-5ms column $(0.25 \mathrm{~mm} \times$ $30 \mathrm{~m} \times 0.25 \mathrm{~mm}$ ) and a 5973 Network Mass Selective Detector (Agilent Technologies). The temperature program used was $60{ }^{\circ} \mathrm{C}$ for $3 \mathrm{~min}, 5{ }^{\circ} \mathrm{C} / \mathrm{min}$ increases up to 120 ${ }^{\circ} \mathrm{C}$, then $20{ }^{\circ} \mathrm{C} / \mathrm{min}$ increases up to $300{ }^{\circ} \mathrm{C}$. Each sample $(1.0 \mathrm{~mL})$ was injected with a run time of $30 \mathrm{~min}$. SCFA concentrations were quantified by comparing their peak areas with standards.

\section{SAG-gel processing step 1: single bacterial cell isolation with gel beads}

Microfluidic droplet generators were fabricated and used for in-bead bacterial genome amplification sequencing. After homogenization of mouse feces in PBS, the supernatant was recovered by centrifugation at $2000 \times g$ for $2 \mathrm{~s}$, followed by $15,000 \times g$ for $3 \mathrm{~min}$. The resulting cell pellets were suspended in PBS, filtered through $35-\mu \mathrm{m}$ nylon mesh, washed twice, and resuspended in $1.5 \%$ agarose in Dulbecco's PBS (DPBS; Thermo Fisher Scientific). 
Prior to single-cell encapsulation, cell suspensions were adjusted to 0.1 cells/bead to prevent the encapsulation of multiple cells in single beads. Using the droplet generator, single microbial cells were encapsulated in droplets and collected in a $1.5-\mathrm{mL}$ tube, which was chilled on ice for $15 \mathrm{~min}$ to form the gel matrix. After solidification, collected droplets were broken with $1 \mathrm{H}$, $1 \mathrm{H}, 2 \mathrm{H}, 2 \mathrm{H}$-perfluoro-1-octanol to collect the beads. Then, the gel beads were washed with $500 \mu \mathrm{L}$ acetone (Sigma), and the solution was mixed vigorously and centrifuged. The acetone supernatant was removed, and 500 $\mu \mathrm{L}$ isopropanol (Sigma) was added, and the solution was mixed vigorously and centrifuged. The isopropanol supernatant was removed, and the gel beads were washed three times with $500 \mu \mathrm{L}$ DPBS.

\section{SAG-gel processing step 2: massively parallel single-cell genome amplification}

Next, individual cells in beads were lysed by submerging the gel beads in lysis solutions: first, $50 \mathrm{U} / \mu \mathrm{L}$ Ready-lyse Lysozyme Solution (Epicentre), $2 \mathrm{U} / \mathrm{mL}$ Zymolyase (Zymo research), $22 \mathrm{U} / \mathrm{mL}$ lysostaphin (MERCK), and $250 \mathrm{U} / \mathrm{mL}$ mutanolysin (MERCK) in DPBS at $37{ }^{\circ} \mathrm{C}$ overnight; second, $0.5 \mathrm{mg} / \mathrm{mL}$ achromopeptidase (MERCK) in DPBS at $37^{\circ} \mathrm{C}$ for $8 \mathrm{~h}$; and third, $1 \mathrm{mg} / \mathrm{mL}$ Proteinase K (Promega) with $0.5 \%$ SDS in DPBS at $40{ }^{\circ} \mathrm{C}$ overnight. At each reagent replacement step, the gel beads were washed three times with DPBS and then resuspended in the next solution. After lysis, the gel beads were washed with DPBS five times and the supernatant was removed. Then, the beads were suspended in Buffer D2 and subjected to multiple displacement amplification using a REPLI-g Single Cell Kit (QIAGEN).

After WGA at $30{ }^{\circ} \mathrm{C}$ for $2 \mathrm{~h}$ and $65{ }^{\circ} \mathrm{C}$ for $3 \mathrm{~min}$, the gel beads were washed three times with $500 \mu \mathrm{L}$ DPBS. Then, the beads were stained with $1 \times$ SYBR Green in DPBS. After confirming DNA amplification by the presence of green fluorescence in the gel, fluorescencepositive beads were sorted into $0.8 \mu \mathrm{L}$ DPBS in 96-well plates by a FACSMelody cell sorter (BD Bioscience) equipped with a 488-nm excitation laser. Green fluorescence intensities were detected through a 550-nm longpass dichroic filter and a 525-nm band-pass filter using fluorescence detector 1 (FL1) with a PMT voltage of 650 and a logarithmic gain. The sample flow rate was adjusted to approximately 200 events/s. At least 5000 particles were analyzed for each histogram. After droplet sorting, 96-well plates proceeded through a second round of WGA or were stored at $-30{ }^{\circ} \mathrm{C}$.

SAG-gel processing step 3: multiplex single-cell genome sequencing

After gel bead collection in 96-well plates, second-round MDA was performed with the REPLI-g Single Cell Kit.
Buffer D2 $(0.6 \mu \mathrm{L})$ was added to each well and incubated at $65{ }^{\circ} \mathrm{C}$ for $10 \mathrm{~min}$. Then, $8.6 \mu \mathrm{L}$ of MDA mixture was added and incubated at $30{ }^{\circ} \mathrm{C}$ for $120 \mathrm{~min}$. The MDA reaction was terminated by heating at $65{ }^{\circ} \mathrm{C}$ for $3 \mathrm{~min}$. After second-round amplification, master library plates of single amplified genomes (SAGs) were prepared. For quality control, aliquots of the SAGs were transferred to replica plates, which were used for DNA yield quantification using a Qubit dsDNA High Sensitivity Assay Kit (Thermo Fisher Scientific) and 16S rRNA gene Sanger sequencing (FASMAC) with the V3-V4 primers described above. Based on the DNA amount and $16 \mathrm{~S}$ rRNA gene sequence, after removing the contaminated samples, to perform whole-genome sequencing, we screened the SAG samples that had enough DNA for library preparation with qualified $16 \mathrm{~S}$ rRNA sequences obtained by Sanger sequencing (more than 400 bp and HQ 40\% were assessed using the Geneious software (Biomatters, Ltd.)). For sequencing analysis, Illumina libraries were prepared using amplicons from the secondround MDA product, using the Nextera XT DNA Library Prep Kit (Illumina) according to the manufacturer's instructions. Each SAG library was sequenced using an Illumina MiSeq $2 \times 75$ bp or HiSeq $2 \times 150 \mathrm{bp}$ configuration (GENEWIZ).

\section{Single-cell genome sequencing analysis}

The sequence reads were assembled de novo using SPAdes 3.9.0 (options: --sc --careful --disable-rr) [39], and the contigs were qualified by QUAST 4.5 [40]. CheckM 1.0.6 [41] with the "lineage_wf" command was used to assess the completeness and the contamination read rate after the contigs smaller than $1000 \mathrm{bp}$ were removed. Composite draft genomes were constructed by co-assembly of SAG groups with $\geq 99 \%$ identical marker genes detected by CheckM and $\geq 95 \%$ ANI. When an assembled composite draft genome showed $\geq 10 \%$ completeness, the composite draft was reassembled from the same SAG group by ccSAG [23]. CDS, rRNAs, and tRNAs were extracted from SAG contigs or composite draft genomes by prokka 1.13 [42], and the metabolic functions were estimated by Genomaple 2.3.2 [43]. Taxonomic annotation of draft genomes was performed by GTDB-Tk 0.3.0, [25], and Bacteroides phylogenetic trees were constructed by the neighbor-joining method using concatenated marker genes detected by CheckM. PUL clusters of Bacteroides composite genomes were detected by BLASTP [44] searches against the Bo cluster with default parameters, and phylogenetic trees were constructed from nucleotide sequences of the PUL clusters in the same manner. Orthologous groups were inferred by OrthoFinder 2.3.3 [45] for additional evaluation of genomic similarities among Bacteroides strains. 


\section{Structure prediction}

Homology modeling of proteins encoded by each gene included in the Bacteroides PUL cluster was performed using SWISS-MODEL [46] in automated mode. Based on the predicted protein structure, structural alignment was performed using MATRAS [47]. SusC-SusD complex structure construction was performed by MODELLER 9.20 [48] using template search and construction scripts created by HOMCOS [49] and using the crystal structure of BT1762-1763 (PDB_5T4Y) as a template.

\section{Supplementary information}

Supplementary information accompanies this paper at https://doi.org/10. 1186/s40168-019-0779-2

Additional file 1: Table S1. Statistics for 346 SAGs obtained from the mouse fecal microbiome Table S2. Taxonomic annotation for 346 SAGs and 28 SAGCs obtained from the mouse fecal microbiome Table S3. Comparison of OTU detection between 16S rRNA gene amplicon sequencing and single-cell sequencing Table S4. Statistics for 28 composite SAGs (IMSAGCs) obtained from the mouse fecal microbiome. Table S5. Detailed statistics for medium and high-quality draft genomes obtained from the mouse fecal microbiome Table S6. Statistics for Bacteroides genomes used in comparative genome analysis Table S7. Numbers of signal peptide-containing predicted glycoside hydrolases/ polysaccharide lyases Table S8. Statistics for high completeness SAGs (> 80\%) associated with inulin uptake and TCA cycle-mediated metabolism Table S9. Statistics for conserved metabolic modules in qualified draft genomes and known Bacteroides spp. reference genomes.

Additional file 2: Figure S1. Comparison of abundance profiles of IMSAGC_001 (OTU2) and IMSAGC_004 (OTU4) in 16S rRNA gene sequencing (a), (b) Violin plots indicate abundance profiles of IMSAGC_001 (OTU2) (a) and IMSAGC_004 (OTU4) (b) before and after two weeks of inulin or cellulose feeding in Lot 1 mice $(n=5$, Tukey's HSD test). (c) Time-dependent changes in abundance profiles of IMSAGC_001 (OTU2) and IMSAGC_004 during 2-weeks inulin feeding in Lot 2 mice $(n=9)$. Figure S2. Predicted structures of sus homologs in IMSAGC_001 and IMSAGC_004 (a) Representation of the predicted SusC-SusD complex protein structure in IMSAGC 001. Units are shown in different colors. The crystal structure of BT1762-1763 (PDB_5T4Y) in Bacteroides thetaiotaomicron was used as template. (b), (c) Trees based on structural alignments of SusC (b) and SusD (c) among fructan-utilizing Bacteroides strains. Figure S3. Predicted partial reverse TCA cycles in IMSAGC 001 and IMSAGC 004 Succinate is formed by the reversal of partial TCA cycle reactions. Pyruvate is carboxylated to form oxaloacetate, which is then reduced to malate, fumarate, and succinate.

\section{Acknowledgements}

The super-computing resource was provided by the Human Genome Center (University of Tokyo).

\section{Authors' contributions}

$\mathrm{MH}, \mathrm{RC}$, and $\mathrm{HT}$ conceived and designed the experiments. MH, YN, and KM developed the SAG-gel workflow. RC conducted animal experiments, metagenomic analysis, and metabolomic analysis. $\mathrm{MH}, \mathrm{YN}, \mathrm{MK}, \mathrm{CS}$, and $\mathrm{KT}$ conducted single-cell genomics experiments and collected the data. $\mathrm{MH}, \mathrm{RC}, \mathrm{MK}$, and $\mathrm{KI}$ conducted bioinformatic analysis of the metagenomic data and single-cell genomic data. MH, RC, MK, Kl, and HT wrote the manuscript. All authors read and approved the final manuscript.

\section{Funding}

This work was supported by JST-PRESTO grant number JPMJPR15FA, JSTCREST Grant Number JPMJCR12A4, MEXT KAKENHI grant numbers 18H01801 and 17H06158, and Cabinet Office, Government of Japan, Cross-ministerial Strategic Innovation Promotion Program (SIP), "Technologies for creating next-generation agriculture, forestry and fisheries"(funding agency: Biooriented Technology Research Advancement Institution, NARO).

\section{Availability of data and materials}

Sequence raw data obtained from mouse gut microbes and high- and medium quality draft genomes are listed in Table 1 and Additional file1: Data from Table S5 were deposited in the DNA Data Bank of Japan (DDBJ) under the accession number PRJDB8805.

\section{Ethics approval and consent to participate}

The protocols of all animal studies were approved by the Committee for Animal Experimentation of the School of Science and Engineering, Waseda University (No. 2017-A057 and 2018-A068)

\section{Consent for publication}

Not applicable.

\section{Competing interests}

M.H. and H.T. are shareholders in bitBiome, to which the patents pertaining to the SAG-gel workflow were transferred.

\section{Author details}

${ }^{1}$ Department of Life Science and Medical Bioscience, Graduate School of Advanced Science and Engineering, Waseda University, 2-2 Wakamatsucho, Shinjuku-ku, Tokyo 162-8480, Japan. ${ }^{2}$ Computational Bio Big-Data Open Innovation Laboratory (CBBD-OIL), National Institute of Advanced Industrial Science and Technology, 3-4-1 Okubo, Shinjuku-ku, Tokyo 169-8555, Japan. ${ }^{3}$ Research Organization for Nano and Life Innovation, Waseda University, 513 Wasedatsurumaki-cho, Shinjuku-ku, Tokyo 162-0041, Japan. ${ }^{4}$ Institute for Advanced Research of Biosystem Dynamics, Waseda Research Institute for Science and Engineering, Graduate School of Advanced Science and Engineering, Waseda University, 3-4-1 Okubo, Shinjuku-ku, Tokyo 169-8555, Japan. ${ }^{5}$ PRESTO, Japan Science and Technology Agency (JST), 5-3 Yonban-cho, Chiyoda-ku, Tokyo 102-0075, Japan.

Received: 27 September 2019 Accepted: 23 December 2019

Published online: 23 January 2020

\section{References}

1. Neis EP, Dejong $\mathrm{CH}$, Rensen SS. The role of microbial amino acid metabolism in host metabolism. Nutrients. 2015;7:2930-46.

2. Human Microbiome Project C. Structure, function and diversity of the healthy human microbiome. Nature. 2012:486:207-14.

3. Macia L, Tan J, Vieira AT, Leach K, Stanley D, Luong S, Maruya M, lan McKenzie C, Hiijikata A, Wong C, et al. Metabolite-sensing receptors GPR43 and GPR109A facilitate dietary fibre-induced gut homeostasis through regulation of the inflammasome. Nat Commun. 2015:6:6734.

4. Poeker SA, Geirnaert A, Berchtold L, Greppi A, Krych L, Steinert RE, de Wouters T, Lacroix C. Understanding the prebiotic potential of different dietary fibers using an in vitro continuous adult fermentation model (PolyFermS). Sci Rep. 2018;8:4318.

5. Kovatcheva-Datchary P, Shoaie S, Lee S, Wahlstrom A, Nookaew I, Hallen A, Perkins R, Nielsen J, Backhed F. Simplified Intestinal Microbiota to Study Microbe-Diet-Host Interactions in a Mouse Model. Cell Rep. 2019:26:3772-83 e6.

6. Schroeder BO, Birchenough GMH, Stahlman M, Arike L, Johansson MEV, Hansson GC, Backhed F. Bifidobacteria or Fiber Protects against DietInduced Microbiota-Mediated Colonic Mucus Deterioration. Cell Host Microbe. 2018:23:27-40 e7.

7. Rakoff-Nahoum S, Coyne MJ, Comstock LE. An ecological network of polysaccharide utilization among human intestinal symbionts. Curr Biol. 2014;24:40-9.

8. Rakoff-Nahoum S, Foster KR, Comstock LE. The evolution of cooperation within the gut microbiota. Nature. 2016:533:255-9.

9. Bolam DN, Sonnenburg JL. Mechanistic insight into polysaccharide use within the intestinal microbiota. Gut Microbes. 2011:2:86-90.

10. Vandeputte D, Falony G, Vieira-Silva S, Wang J, Sailer M, Theis S, Verbeke K, Raes J. Prebiotic inulin-type fructans induce specific changes in the human gut microbiota. Gut. 2017;66:1968-74

11. Sonnenburg ED, Zheng H, Joglekar P, Higginbottom SK, Firbank SJ, Bolam DN, Sonnenburg JL. Specificity of polysaccharide use in intestinal 
bacteroides species determines diet-induced microbiota alterations. Cell. 2010;141:1241-52.

12. Catry E, Bindels LB, Tailleux A, Lestavel S, Neyrinck AM, Goossens JF, Lobysheva I, Plovier H, Essaghir A, Demoulin JB, et al. Targeting the gut microbiota with inulin-type fructans: preclinical demonstration of a novel approach in the management of endothelial dysfunction. Gut. 2018;67:27183.

13. Langille MG, Zaneveld J, Caporaso JG, McDonald D, Knights D, Reyes JA, Clemente JC, Burkepile DE, Vega Thurber RL, Knight R, et al. Predictive functional profiling of microbial communities using 165 rRNA marker gene sequences. Nat Biotechnol. 2013;31:814-21.

14. Scholz M, Ward DV, Pasolli E, Tolio T, Zolfo M, Asnicar F, Truong DT, Tett A Morrow AL, Segata N. Strain-level microbial epidemiology and population genomics from shotgun metagenomics. Nat Methods. 2016;13:435-8.

15. Pasolli E, Asnicar F, Manara S, Zolfo M, Karcher N, Armanini F, Beghini F, Manghi $P$, Tett A, Ghensi $P$, et al. Extensive unexplored human microbiome diversity revealed by over 150,000 genomes from metagenomes spanning age, geography, and lifestyle. Cell. 2019;176:649-62 e20.

16. Almeida A, Mitchell AL, Boland M, Forster SC, Gloor GB, Tarkowska A, Lawley TD. Finn RD: a new genomic blueprint of the human gut microbiota. Nature. 2019;568:499-504.

17. Nayfach S, Shi ZJ, Seshadri R, Pollard KS, Kyrpides NC. New insights from uncultivated genomes of the global human gut microbiome. Nature. 2019; 568:505-10.

18. Thaiss CA, Zeevi D, Levy M, Zilberman-Schapira G, Suez J, Tengeler AC, Abramson L, Katz MN, Korem T, Zmora N, et al. Transkingdom control of microbiota diurnal oscillations promotes metabolic homeostasis. Cell. 2014; 159:514-29.

19. Thaiss CA, Levy M, Korem T, Dohnalova L, Shapiro H, Jaitin DA, David E, Winter DR, Gury-BenAri M, Tatirovsky E, et al. Microbiota diurnal rhythmicity programs host transcriptome oscillations. Cell. 2016;167:1495-510 e12.

20. Segata N, Izard J, Waldron L, Gevers D, Miropolsky L, Garrett WS Huttenhower C. Metagenomic biomarker discovery and explanation. Genome Biol. 2011;12:R60.

21. Zhang M, Zhang Y, Scheuring CF, Wu CC, Dong JJ, Zhang HB. Preparation of megabase-sized DNA from a variety of organisms using the nuclei method for advanced genomics research. Nat Protoc. 2012;7:467-78.

22. Hosokawa M, Nishikawa $Y$, Kogawa M, Takeyama H. Massively parallel whole genome amplification for single-cell sequencing using droplet microfluidics. Sci Rep. 2017;7:5199.

23. Kogawa M, Hosokawa M, Nishikawa Y, Mori K, Takeyama H. Obtaining highquality draft genomes from uncultured microbes by cleaning and coassembly of single-cell amplified genomes. Sci Rep. 2018;8:2059.

24. Bowers RM, Kyrpides NC, Stepanauskas R, Harmon-Smith M, Doud D, Reddy TBK, Schulz F, Jarett J, Rivers AR, Eloe-Fadrosh EA, et al. Minimum information about a single amplified genome (MISAG) and a metagenomeassembled genome (MIMAG) of bacteria and archaea. Nat Biotechnol. 2017; 35:725-31.

25. Parks DH, Chuvochina M, Waite DW, Rinke C, Skarshewski A, Chaumeil PA, Hugenholtz P. A standardized bacterial taxonomy based on genome phylogeny substantially revises the tree of life. Nat Biotechnol. 2018;36:996-1004.

26. Glenwright AJ, Pothula KR, Bhamidimarri SP, Chorev DS, Basle A, Firbank SJ, Zheng $\mathrm{H}$, Robinson CV, Winterhalter M, Kleinekathofer U, et al. Structural basis for nutrient acquisition by dominant members of the human gut microbiota. Nature. 2017;541:407-11.

27. Spiga L, Winter MG, Furtado de Carvalho T, Zhu W, Hughes ER, Gillis CC, Behrendt CL, Kim J, Chessa D, Andrews-Polymenis HL, et al. An oxidative central metabolism enables salmonella to utilize microbiota-derived succinate. Cell Host Microbe. 2017;22:291-301 e6.

28. Connors J, Dawe N, Van Limbergen J. The role of succinate in the regulation of intestinal inflammation. Nutrients. 2018;11.

29. Lan F, Demaree B, Ahmed N, Abate AR. Single-cell genome sequencing at ultra-high-throughput with microfluidic droplet barcoding. Nat Biotechnol. 2017;35:640-6.

30. Xu L, Brito IL, Alm EJ, Blainey PC. Virtual microfluidics for digital quantification and single-cell sequencing. Nat Methods. 2016;13:759-62.

31. Rinke C, Schwientek P, Sczyrba A, Ivanova NN, Anderson IJ, Cheng JF, Darling A, Malfatti S, Swan BK, Gies EA, et al. Insights into the phylogeny and coding potential of microbial dark matter. Nature. 2013;499:431-7.

32. Rinke C, Lee J, Nath N, Goudeau D, Thompson B, Poulton N, Dmitrieff E, Malmstrom R, Stepanauskas R, Woyke T. Obtaining genomes from uncultivated environmental microorganisms using FACS-based single-cell genomics. Nat Protoc. 2014;9:1038-48.

33. Miyamoto $Y$, Itoh K. Bacteroides acidifaciens sp. nov., isolated from the caecum of mice. Int J Syst Evol Microbiol. 2000;50(Pt 1):145-8.

34. Bolyen E, Rideout JR, Dillon MR, Bokulich NA, Abnet CC, Al-Ghalith GA, Alexander $\mathrm{H}$, Alm EJ, Arumugam M, Asnicar F, et al. Reproducible, interactive, scalable and extensible microbiome data science using QIIME 2. Nat Biotechnol. 2019;37:852-7.

35. Callahan BJ, McMurdie PJ, Rosen MJ, Han AW, Johnson AJ, Holmes SP. DADA2: High-resolution sample inference from Illumina amplicon data. Nat Methods. 2016:13:581-3.

36. Quast C, Pruesse E, Yilmaz P, Gerken J, Schweer T, Yarza P, Peplies J, Glockner FO. The SILVA ribosomal RNA gene database project: improved data processing and web-based tools. Nucleic Acids Res. 2013;41:D590-6.

37. Edgar RC. Search and clustering orders of magnitude faster than BLAST. Bioinformatics. 2010;26:2460-1.

38. Edgar RC. UPARSE: highly accurate OTU sequences from microbial amplicon reads. Nat Methods. 2013;10:996-8.

39. Bankevich A, Nurk S, Antipov D, Gurevich AA, Dvorkin M, Kulikov AS, Lesin VM, Nikolenko SI, Pham S, Prjibelski AD, et al. SPAdes: a new genome assembly algorithm and its applications to single-cell sequencing. J Comput Biol. 2012;19:455-77.

40. Gurevich A, Saveliev V, Vyahhi N, Tesler G. QUAST: quality assessment tool for genome assemblies. Bioinformatics. 2013;29:1072-5.

41. Parks DH, Imelfort M, Skennerton CT, Hugenholtz P, Tyson GW. CheckM: assessing the quality of microbial genomes recovered from isolates, single cells, and metagenomes. Genome Res. 2015;25:1043-55.

42. Seemann T. Prokka: rapid prokaryotic genome annotation. Bioinformatics. 2014;30:2068-9.

43. Arai W, Taniguchi T, Goto S, Moriya Y, Uehara H, Takemoto K, Ogata H, Takami H. MAPLE 2.3.0: an improved system for evaluating the functionomes of genomes and metagenomes. Biosci Biotechnol Biochem. 2018:82:1515-7.

44. Altschul SF, Gish W, Miller W, Myers EW, Lipman DJ. Basic local alignment search tool. J Mol Biol. 1990;215:403-10.

45. Emms DM, Kelly S. OrthoFinder: solving fundamental biases in whole genome comparisons dramatically improves orthogroup inference accuracy. Genome Biol. 2015;16:157.

46. Schwede T, Kopp J, Guex N, Peitsch MC. SWISS-MODEL: an automated protein homology-modeling server. Nucleic Acids Res. 2003;31:3381-5.

47. Kawabata T. MATRAS: A program for protein 3D structure comparison. Nucleic Acids Res. 2003:31:3367-9.

48. Marti-Renom MA, Stuart AC, Fiser A, Sanchez R, Melo F, Sali A. Comparative protein structure modeling of genes and genomes. Annu Rev Biophys Biomol Struct. 2000;29:291-325.

49. Fukuhara N, Kawabata T. HOMCOS: a server to predict interacting protein pairs and interacting sites by homology modeling of complex structures. Nucleic Acids Res. 2008;36:W185-9.

\section{Publisher's Note}

Springer Nature remains neutral with regard to jurisdictional claims in published maps and institutional affiliations.

Ready to submit your research? Choose BMC and benefit from:

- fast, convenient online submission

- thorough peer review by experienced researchers in your field

- rapid publication on acceptance

- support for research data, including large and complex data types

- gold Open Access which fosters wider collaboration and increased citations

- maximum visibility for your research: over $100 \mathrm{M}$ website views per year

At $\mathrm{BMC}$, research is always in progress.

Learn more biomedcentral.com/submission 